\title{
TRAUMATIC OCCLUSION.
}

\author{
By Paul R. Stillman, D. D. S., New York City, N. Y.
}

(Read before the National Dental Association at Its Trenty-second Annual Session, Chicago, Ill., August 5-9, 1918.)

$\mathrm{T}$ $\mathrm{HE}$ term occlusion as used in dentistry defines the mutual mechanical relations of the opposing teeth. The word trauma means an injury caused by force. Thus the combination of these two words describes an injury by force which is caused by improper mechanical relations of the teeth during function.

The accepted definition of Traumatic Occlusion is as follows: "A perversion of the occlusal relations of the teeth when in use, whereby an excessive stress is brought upon one or more antagonizing teeth."

When Angle defined the term malocclusion reference was only to the passive arrangement of the teeth as they would appear from an examination of casts. The definition of Angle reads: "A perversion of the normal relations of the teeth when the jaws are closed." If these relations are perfectly normal in every way when the jaws are closed, they will also be normal when the jaws are used in mastication. Therefore in order to be completely satisfactory, this definition must be understood to imply that the relations of the teeth shall be so perfect as to be normal also in the functions of mastication as well as when occluding, a point which is very apt to be overlooked by the average observer in studying post mortem osteology or making orthodontic diagnosis from casts. .

The Angle definition does not, on the face of it, take into consideration the axial measurements of the teeth as in supra and infra-occlusion nor their relation in the condyle path to the plane of occlusion, nor does it include the relation of the associated structures or attempt to define that greatest of all the necessities in occlusion viz coordination in function. Neither will this paper attempt to solve this problem. All that this paper hopes to accomplish is to call attention to a fact which has probably been frequently observed by many others, that there is a mal-relation of the teeth in function-a relation which satisfies the definition of normal occlusion as defined by Angle-yet, in which there exists incoordination of the occlusal inclined planes during the function of mastication. In other words Traumatic Occlusion may co-exist with apparent normal occlusion in both those cases in which the arches have developed in the natural way as well as those which have been treated according to the present day standards of orthodontic practice. Several finished or completed cases in orthodontia have been observed in which the occlusal re-arrangement of the teeth presented to the not too critical observer, a very satisfactory as well as a very esthetic appearance, where the departure from the ideal as established by the rule of the Angle classification would appear as insignificant. To the periodontist these cases have shown symptoms of incipient periodontal disease, gingivitis and the presence of 
detritus. Cases which persistently show these symptoms are suspected of having a traumatic occlusion and traumatic occlusion has, in fact, been found in all of these cases. When they were referred back to the orthodontists who had treated the mal-occlusion, appliances were replaced and treatment for coordination of the occlusion instituted.

Normal occlusion is usually accompanied by co-existing normal co-ordination in function. 'This has never been denied. Normal occlusion in certain skull specimens, (such for instance as the well known Broomell specimen), shows an amazing absence of periodontoclasia when examined by a periodontist, while at the same time it excites the admiration of orthodontists for the perfection of its arch arrangement and its ideal plane of occlusion.

The American Academy of Periodontology, thru its Committee on Scientific Research and Nomenclature, has adopted a statement of the following conditions essential to normality in the peridontal tissues and also in regard to the occlusal relations predisposing to traumatic occlusion, and I cannot do better than to interpolate two statements: First: conditions essential to normality in the periodontal tissues from the standpoint of occlusion.

1. Normal mesio-distal and buccolingual relationship between the jaws and between the individual teeth.

2. Normal distance or space between the jaws vertically and laterally or individual opposing teeth when the teeth are in occlusion.

3. Normal occlusal surfaces (either natural or restored) with proper coordination of these with the opposing jaw.

4. Normal contact points, natural or restored, between all teeth.

5. Wear of the inclined planes of the cusps thru use in mastication, thereby bringing about a dissipation of (oc- clusal) bucco-lingual stress, in the gliding motion of tooth against tooth. Second: Occlusal relations predisposing to traumatic occlusion.

1. Normal occlusion (Angle classification) where opposing cusps and inclined planes interfere with freedom of motion between the jaws and individual teeth; in other words, lack of coordination.

2. Mal-Occlusion (Angle) of every form.

3. Shifting thru lost approximal contact support due to extraction.

4. Faulty restorations, including fillings, crowns, bridges, fixed or removable, where inco-ordination exists between them and the opposing teeth, insufficient number or strength of abutments, etc.

Traumatic occlusion may be acquired when any of the teeth are driven outside their normal limits of motion within their sockets thru excessive occlusal pressure. These improper occlusal relations may be produced by shifting-.. as where a first or second molar has been lost: instance, a shifting forward of the second and third molars into the space recently occupied by a first molar. It may be produced by the erupting of second molars in torsion; orthodontists observe in this a potent force for mal-adjustment and it is likewise doubtless a potent factor in the etiology of traumatic occlusion. Faulty contact points: instance, in all approximal restorations by plastic materials where the contact point is lost by a lack of integrity of the material: instance careless operations of ignorant operators. Occlusal operations: instance, when an occlusal surface of either a filling or a crown is built too high either on one or more cusps, or where the absence of cusp formation permits a shifting of the tooth thus distributing force even to the entire arch. Formation of abnormal habits in masti- 
cation: instance, when the habit is to use one side to the exclusion of the other, resulting in an absence of wear upon the side which is in disuse. Again the deformity caused by abrasion of the inclined planes may so interfere with function as to produce stress when the dentures are worn into each other. In such cases as this where the bite has been slightly closed thru occlusal wear of the bicuspids and molars the cuspids and incisors also suffer thru a lack of wear.

One may conclude that "health depends upon normal function," deformity either of the teeth themselves or of the relation of the teeth in the arches interferes with function. Traumatic occlusion is the sequal of dental inco-ordination.

Nearly every writer upon the subject of periodontal disease has recognized mal-occlusion as an etiological factor in its development. Yet, strange to say, the particular form of mal-occlusion with which we are dealing has only been identified and recognized as a potent factor in the causation of periodontal disease in recent years.

The first principle of the treatment of any disease is an attempt to eliminate the contributory factors. This being the case, does it not appear to be more than an accident of fate which has brought the two specialties of periodontia and orthodontia together into one department of the National Dental Association? Was it not an act of Providence?

Analysis of cases indicates that occlusal trauma is manifested in three ways as regards the application of force to the tooth. First: thru elongation, whereby the tooth extends out of its socket, Second: thru tipping, whereby force is applied at an abnormal angle to the long axis of the tooth. Third: the wedging of one inclined plane in gliding past another, whereby the tooth is forced to one side of its socket.

Study of these cases reveals the fact that the injury is of a different character from that produced by any other etiological factor. The impact of a traumatic occlusion is transmitted thru the tooth structure to the entire pericementum and associated structures instead of first attacking the tissues at the gingival border, as is the case with all other irritants. This accounts for the character of the lesions produced, most noticeable among which are excessive mobility of the tooth and pocket formation.

Traumatic occlusion unless compensated by abrasion thru the aid of vigorous general and local health, invariably exhibits accompanying symptoms of periclasia. This stress when sufficiently pronounced may induce a lesion of the pericemental tissues, which in its early stages is physically manifested by an abnormal mobility of the teeth and radiographically by a thickened or hypertrophied pericementum and radiolucent areas in the osseous investment. The macroscopic incipient symptoms may be no more than capillary stasis of the gingival and organ circulation; while those in which the condition has been allowed to continue over a period of years may present the familiar atrophic symptoms of a chronic so-called pyorrhea.

The detection of the traumatic factor in advanced cases of dental periclasia is comparatively simple. Socket mobility and pocket formation point unerringly to traumatic occlusion. Before these lesions have been produced we must look for milder symptoms. Gingivitis and serumal calculus are early symptoms, especially when these are not readily explainable by the presence of other etiological factors. Peculiar elevations of tissue usually of the same color as the gingival tissue, a little above the 
margin and dark lines running from the gingival margin parallel to the tooth root are also some of the diagnostic signs.

The treatment of all of these cases may best be accomplished by orthodontic procedure and in the youthful it is easy to procure orthontic treatment. But many of the cases of mal-occlusion, in fact the largest percentage of cases in the practice of a periodontist, are pathological being complicated with periodontoclasia and are past the age of youth, most of them occuring in middle or in advanced middle life. Upon this type of case the average orthodontist proceeds to frown. There is a very general belief among these specialists that cases pathological which have passed the period of youth are not desirable cases. Doubtless they present problems and phazes in treatment which are difficult and at the present time not widely understood. Yet here is a field in orthodontic practice which opens up an almost limitless supply of clinical material as well as a very interesting subject for study.

Every case of traumatic occlusion needs functional or physiological rest for the peridontal tissue cells before the benign processes of cell regeneration may be inaugurated. This rest may be obtained by co-ordinating the occlusal surfaces thru modifying their shape. This is usually accomplished by grinding, a method of treatment which has already been described. It is a method which could not be considered other than pernicious by the average orthodontist of today. The very fundamentals of orthodontic teaching and practice are violated when teeth are mutilated, provided their form was previously normal. Yet it is necessary in surgery at times to deform and mutilate in order to save life. So it is in periodontia and even at times in orthodontia. Nature deforms by function. The orthodontist who is treating a case of adult mal-occlusion and who finds it difficult to secure an ideal cusp relationship either because of acquired deformity thru occlusal abrasive wear or a missing tooth, must make up his mind that it is necessary to grind the cusps during treatment so that an arrangement may be secured which will give co-ordination in the functions of closing and mastication. This is only what nature herself does by abrasion if the supporting tissues are sufficiently vigorous. Lacking this, traumatic occlusion is instituted and disease will surely follow.

The peridontist's cases are always pathological while those of the orthodontist are usually physiological. Unless the orthodontist steps into the breach with the special knowledge he possesses upon the subject of occlusion, the mutilation of teeth by grinding must continue to be practiced for the reason that, thru these admittedly inferior methods, even some of the most advanced cases of periclasia are restored to periodontal health.

\section{Discussion.}

\section{J. Herbert Hood, Cleveland, Ohio.}

I believe that the presentation of the subject of traumatic occlusion is most opportune at this time. I regret very much that Doctor Stillman could not have been with us to present the subject in person, as he could undoubtedly have brought out in the discussion many important points not mentioned in his paper. However, Doctor Hayden has presented the paper in a most admirable way.

I have had the privilege of discussing this subject with Doctor Stillman upon divers occasions and my views, based upon my experience and observation, coincide closely with his. The essayist makes the claim that when any tooth is malapposed to the extent that the occlusal planes of the antagonizing teeth do not come into apposition in the normal manner, that undue stress is pro- 
duced and that the tooth is $\mathrm{c}$ : $r$-functioned, as it were, during mas ication and that incipient peridontoclasia may be produced. The extent of the disease will be in proportion as abnormal stress is placed upon the malapposed teeth. For instance, I have observed a number of cases in which a lower lateral incisor was in labial occlusion. Every time the jaws were closed in the act of masticating a tremendous amount of force was exerted upon the lower teeth, the leverage no doubt being greater in this type of case than would be the case where the tooth was stronger and more firmly imbedded in the alveolar process. The result was that a large amount of tissue was destroved. The fibers of the periodontal membrane became detached and the border of the alveolus resorbed. Upon the restoration of the normal occlusal relations and with proper periodontic treatment the case made an uneventful recovery.

These are typical cases and should be observed more closely by the general practitioner and the specialist in periodontoclasia. There are many other cases which are not so typical and which are more difficult to diagnose that are frequently over-looked in the examination of the mouth. I am of the opinion that the cases which may be logically classified under traumatic occlusion open up a field for investigation, experimentation and treatment that is practicallv unlimited.

We are all very grateful to Doctor Stillman for consenting to present the subject at this time. The arrangement was made with him thru the Council of the Academy, and we were fortunate in having so able a person as Doctor Hayden to present the paper to us in Doctor Stillman's absence.

\section{H. J. Leonard, Minneapolis, Minn.}

This subject of traumatic occlusion is one which coupled with Doctor Howe's paper and contention that metabolic fac- tors are causative of pyorrhea, I believe will go far toward explaining may things about etiology. We have been taught to think of pyorrhea as an infection and invasion of bacteria at certain points breaking down the gum tissue and causing periodontoclasia at that point. We must come to the conclusion from this paper that there is a lowering of resistance due to svstemic mal-metabolism, and mal-occlusion and mal-articulation which goes very far toward explaining cases of pvorrhea. I am very much delighted with this paper and I think it and Doctor Howe's are stepping stones in the treatment of pyorrhea.

\section{L. Rhein, New York City.}

I have been very much interested in Doctor Stillman's work in New York City, and as long as he is ill in New York at present it may be perfectly proper for me to say that we possess in Doctor Stillman an exceptional man in this particular field of work. His absolute professionalism, his honesty and unswerving integrity, as I have known for years are features that are, I am sorry to say, not as ccmmon as they ought to be in professiorsal life.

The question of malocclusion in its relation to periclasia is not a new one to us by any means. We have had a great deal said on this subject in years gone by, but the difficulty has been in determining in regard to these matters what we should consider as the actual etiologic factor in any form of priclasia. Now there is nothing in the whole line of our special field of work over which there has been such a vast amount of misunderstanding, misinterpretation and division of opinion as to these conclusions. We have been filled to repletion at times with the diseases that are caused bv periclasia in its different stages, and all clinical evidence followed up by the proper laboratory research, disproved, everthing of that kind. The general medical practitioner in the great fad of 
the day, mouth infection as related to human health, has not the slightest idea of the differential diagnosis between a focal infection that is concealed in the alveolar structure and a condition in the mouth of superficial suppuration which has an exit in the mouth. It is easy for us to understand that the general medical practitioner should not be capable of making a correct diagnosis of these conditions and generally places the cart before the horse, but the peculiar thing is that a lack of proper scientific foundation in the educational curriculum of dentistry has brought to us a mass of practitioners who make the same error day after day. I have no doubt that the advances we are now having and the prospect of improved courses in our educational institutions will bring about improvement and that the time will come when the dentist gets his diploma that he will know something about how to make a diagnosis in the mouth. No dental college in the past has taught him this. They seem in some peculiar way not to have considered this as being of any importance in the practice of dentistry. In other words, let us put the stamp of disapproval on the usual jumping at conclusions by men who hand themselves out to the general practitioners as specialists in this line. When they have a patient sent to them the first thing they consider is that they have to do something immediately for that patient in order to retain that patient. The idea of understanding what they are to treat is something that has never entered their mind. As I recall it, this paper has gone into some detail on the question of a proper diagnosis of the conditions that exist in the mouth, and a proper understanding of the causes that practically make such an abnormal condition possible. In all my experience I know of no case of any form of malocclusion that per se could possibly produce a condition such as we now speak of as peri- clasia. That these conditions of malocclusion in different grades, especially the kind mentioned by the essayist, have a proven significance in the production of these local evidences of malnutrition is beyond question. That they weaken the parts locally and make them vulnerable to any infective organism is a question beyond argument, but I have failed -and if my knowledge of what the pathological conditions are that exist in any part of the human body as understood by our scientists at the present time is at all correct, it is absolutely impossible for any condition of malocclusion to produce such a thing per se, in the normal human being, and it is dependent upon how much we get away from normality as to the possibility of infection in a distinctive part of the alveolar structure. Some form of malnutrition must be present, and as that condition of malnutrition is more or less expressed in the special case of periodontoclasia, so will the degrees of the different kinds of malnutrition exert a most vital significance on the symptomatology of the suppurative conditions. While on this subject I desire to call attention to the mistaken diagnosis of perioclasia where there is nothing more nor less than undiagnosed cases of alveolar abscess. They frequently come from some distance and perforate their way thru the alveolar process, are diagnosed and treated locally as forms of perioclasia.

The unnecessary treatment and pain with serious injury to the superficial tissues is a menace to the communal health that is steadily increasing in proportion to the increase of incompetent dentists calling themselves "Specialists in Pyorrhea."

What action can this section take that would be a help to our patients?

\section{Frank $M$. Casto, Cleveland, $O$.}

The reading of Dr. Stillman's paper, and the discussion of same have been very interesting to me. Of course I 
regret the fact that Dr. Stillman could not be present. He could have cleared up a number of points in his paper, which are more or less obscure.

The question of Traumatic Occlusion, as proposed by Dr. Stillman, pleases me very much, altho his definition is not entirely clear to me.

I do not quite understand whether or not Dr. Stillman means that in every case where the occlusal incline planes are not in absolutely normal contact it is a case of Traumatic Occlusion. There are certainly many cases in which Traumatic Occlusion may be diagnosed without difficulty. Several instances have been cited here today, but there are other cases in which to my mind there is a grave question as to whether they could be properly diagnosed as Traumatic Occlusion. I believe it is possible to have some abnormality exist in regard to contact of the occlusal incline planes, and still not have what I understand as Traumatism. In these cases there may be no more pressure or force brought to bear upon the particular teeth that are in slight position of Malocclusion that there would be if they were in a normal relation, as for instance the slight rotation of certain of the bicuspid teeth, also in certain instances where the bicuspids may be in a slight lingual or buccal Occlusion. I believe that in any cases where the teeth are in Malocclusion, and where the stress of mastication is brought upon them, with greater force than is normal, the result would be a Traumatic Occlusion, followed by certain pathological conditions.

I certainly appreciate the work that Dr. Stillman has been doing along this line, and the results which he is obtaining are most creditable, and of great value to both the Periodontist and Orthodontist.

I wish to take this opportunity to thank him personally for giving us of his time and knowledge, and presenting this subject here today to the first session of the Section on Orthodontia and Periodontia.

\section{Gillette Hayden, Columbus, Ohio.}

I am sure that if Dr. Sitillman were here he would very greatly appreciate the discussion which has been accorded his paper.

Every periodontist, I think, believes with the first discusser that there must be a lowering of the vitality of the parts surrounding the tooth before there is an invasion of these tissues by microorganisms.

Could Dr. Sitllman have been able to hear Dr. Rhein's very valuable contribution he would say that there is no one etiological factor which is capable of producing dental perioclasia. It is a combination of factors such as traumatic occlusion and the accumulations of soft or hard deposits upon the teeth plus bacteria. No one of these alone will produce periodontoclasia. 